
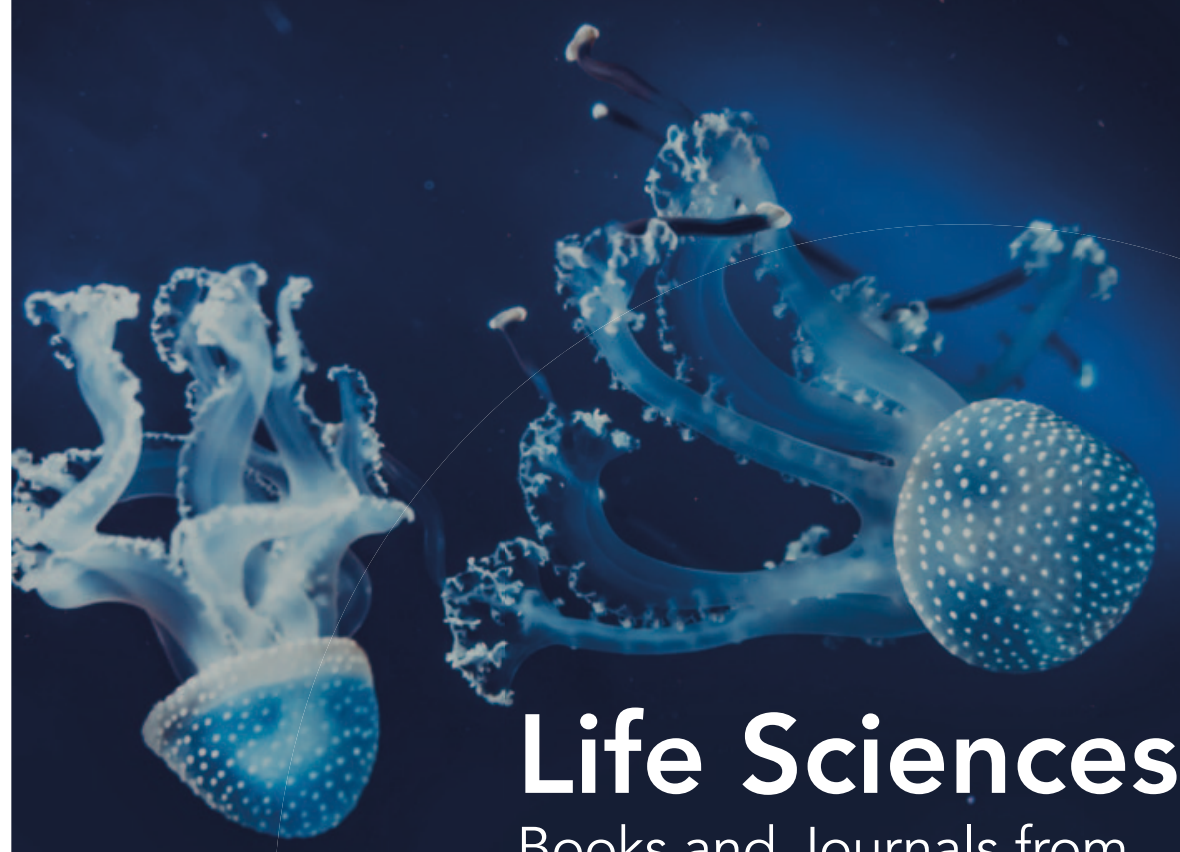

Books and Journals from Cambridge University Press

Cambridge is one of the leading publishers in ecology and conservation biology and publishes high quality texts and research across the breadth of the life sciences, focusing particularly on animal behaviour, biological anthropology, evolutionary biology, computational and systems biology, as well as statistics and professional development titles for biologists.

We also have an extensive portfolio of established journals in agriculture, ecology and conservation, and animal science.

For further details visit: cambridge.org/core-life-sciences

\title{
Çambridge Core
}

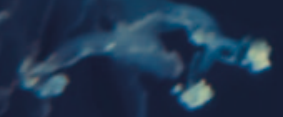




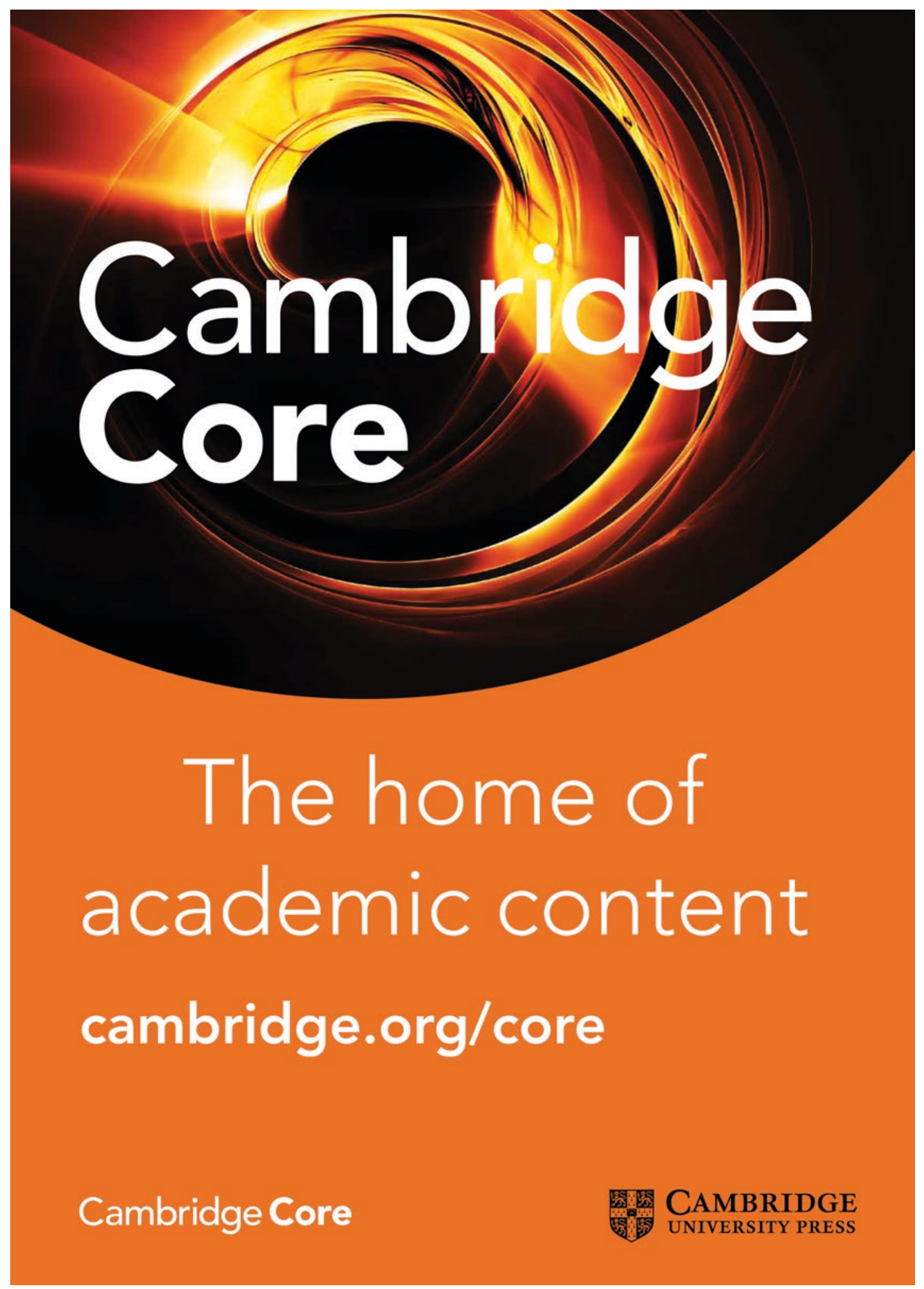




\title{
Entomological Society of Canada / Société d'entomologie du Canada
}

\author{
386 Broadway, Suite 503 \\ Winnipeg, Manitoba, R3C 3R6 Canada \\ www.esc-sec.ca \\ telephone/téléphone 888-821-8387, + 1 204-282-9823; fax/télécopieur +1 204-947-9767; \\ e-mail/courrielinfo@esc-sec.ca
}

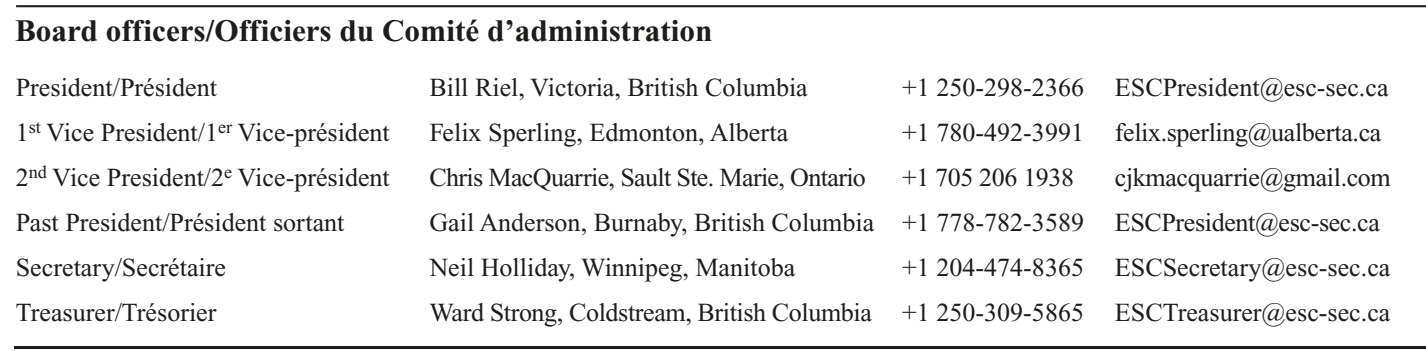

The Entomological Society of Canada was founded in 1863 to study, advance, and promote entomology. It does this through publications, meetings, scholarships and awards, advocacy, and other activities. Membership is open to all individuals interested in entomology worldwide and is renewable annually. Regular members receive the scientific journal of the Society, The Canadian Entomologist, published continuously since 1868, and the newsletter of the Society, the Bulletin. Student members receive the Bulletin and can elect to receive The Canadian Entomologist for a nominal surcharge. All members receive a discount for annual meeting registration fees, have access to members-only privileges at the Society Web site, and receive other benefits. Details on regular and student membership fees and application forms are also available at the Web site. Application forms should be transmitted to the Society Office.

All information found on this page is also available at www.esc-sec.ca. Direct all correspondence about membership, subscriptions, back issues, and changes of address to the Society Office.

Fondée en 1863, la Société d'entomologie du Canada a pour but d'étudier, de faire progresser et de promouvoir l'entomologie. Elle accomplit ceci par des publications, des réunions, des bourses et prix, par plaidoyer ou autres activités. L'adhésion est ouverte à toute personne au monde intéressée à l'entomologie, et elle est renouvelable annuellement. Les membres réguliers reçoivent le journal scientifique de la Société, The Canadian Entomologist, qui a été publié sans interruption depuis 1868, et le Bulletin de la Société. Les membres étudiants reçoivent le Bulletin de la Société et, moyennant un coût nominal, The Canadian Entomologist. Tous les membres ont des rabais pour l'inscription à la réunion annuelle, un accés privilégié à la section des membres du site Internet, ainsi que d'autres advantage. Pour les formulaires d'application et des informations concernant l'adhésion régulière ou étudiante, consultez le site Web. Les formulaires d'adhésion doivent être acheminés au siège de la Société.

Toutes les informations sur cette page sont également disponibles au www.esc-sec.ca. Adressez toute correspondance concernant l'adhésion, les abonnements institutionnels, les numéros passés et les changements d'adresses au siège de la Société. 


\section{Back cover/Quatrième de couverture:}

Samurai wasp, Trissolcus japonicus (Hymenoptera: Scelionidae), parasitising egg of Halyomorpha halys [Delémont, Switzerland]

La guêpe samuraï, Trissolcus japonicus [Hymenoptera : Scelionidae), parasitant des œufs de Halyomorpha halys [Delémont, Suisse]

[Photo: Tim Haye]

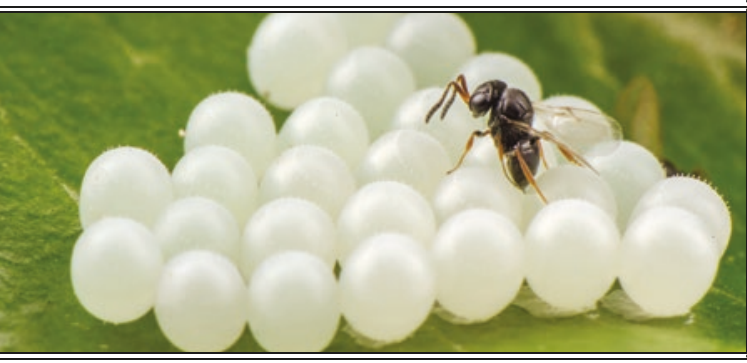

Cambridge Core

For further information on this journal

please go to the journal website at:

cambridge.org/tce

\section{CAMBRIDGE UNIVERSITY PRESS}

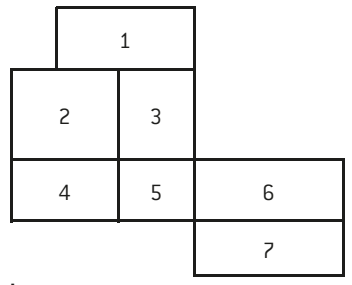

Images

\section{Front cover/Page couverture:}

1. Robber fly in the Subfamily Asilinae (Diptera: Asilidae), photo taken at Trout Creek Poin [Summerland, British Columbia, Canada]

Une mouche à toison de la sous-famille Asilinae (Diptera : Asilidae) photographiée à Trout Creek Point [Summerland, Colombie-Britannique, Canada]

[Photo: Robyn DeYoung]

2. Portrait of a male American rubyspot, Hetaerina americana (Odonata: Calopterygidae), photographed along the Châteauguay River on 13 August 2020. A small population of this colourful damselfly was discovered at this site, well north of its usual range, several years ago [Ste-Martine, Quebec, Canada] Portrait d'un mâle de la courtisane d'Amérique (Hetaerina americana) (Odonata : Calopterygidae) photographié le long de la rivière Châteauguay le 13 août 2020. Une petite population de cette demoiselle colorée a été découverte sur ce site, bien au nord de sa distribution habituelle, il y a quelques années. [Sainte-Martine, Québec, Canada] [Photo: Richard Yank]

3. A female Cyphoderris monstrosa (Orthoptera) found hanging out in a backyard [Summerland, British Columbia, Canada]

Une femelle Cyphoderris monstrosa (Orthoptera) trouvée dans une cour arrière [Summerland, Colombie-Britannique, Canada]

[Photo: Andrea Brauner]

4. Two male rhinoceros beetles (Xylotrupes: Dynastinae) preparing to 'fight.' Prize fighters are bred and bets are placed on which male will throw the other off a cylindrical piece of wood [Chiang Mai, Thailand]

Deux mâles scarabées rhinocéros (Xylotrupes, Dynastinae) se préparant à se « battre ». Les combattants sont issus d'élevage et les paris sont placés sur le mâle qui lancera l'autre sur un morceau de bois cylindrique. [Chiang Mai, Thaïlande]

[Photo: Matt Muzzatti]

5. Subadult female black widow spider (Latrodectus sp.) walking on her web [Tsawwassen, British Columbia, Canada]

Une femelle subadulte de la veuve noire (Latrodectus sp.) marchant sur sa toile. [Tsawwassen, Colombie-Britannique, Canada]

[Photo: Andreas Fischer]

6. Enallagma civile (Odonata: Coenagrionidae) watching the foot traffic along a boardwalk [Riding Mountain National Park, Manitoba, Canada]

Enallagma civile (Odonata : Coenagrionidae) observant la circulation piétonne le long d'un trottoir [Parc national Riding Mountain, Manitoba, Canada]

[Photo: Mel Hart]

7. Collecting insects and setting up pan traps on the dry slopes as part of the Biodiversity Galiano Project [Galiano Island, British Columbia, Canada]

Récolte d'insectes et installation de pièges à interception sur des pentes sèches dans le cadre du projet Biodiversité Galiano [île Galiano, Colombie-Britannique, Canada]

[Photo: Chris Ratzlaff] 